\title{
Propuesta metodológica de iniciación al esquí alpino escolar mediante esquís cortos
}

\author{
Javier CONDE-PIPÓ \\ Blanca ROMÁN \\ Mario FERNÁNDEZ-AGUILERA \\ Félix ZURITA
}

Datos de contacto:

Javier Conde Pipó

Universidad de Granada

javiconde@ugr.correo.es

Blanca Román Alconchel

Colegio Ave María La Quinta

blancaromanalcochel@gmail.com

Mario Fernández Aguilera

Federación Andaluza Deportes

de Invierno

fdez.aguilera@gmail.com

Félix Zurita Ortega

Universidad de Granada

felixzo@ugr.es

\begin{abstract}
RESUMEN
El esquí alpino es un deporte complejo pero con baja tasa de abandono, idóneo para promover la práctica deportiva en edad escolar. Evitar el fracaso y frustración en la iniciación requiere de un método de enseñanza que facilite la progresión. El objetivo de este estudio fue determinar si es eficaz un método basado en el empleo de esquís cortos. La muestra se compuso de 37 sujetos, de entre 8 y 9 años de edad ( $M=8.12 ; \mathrm{DT}=0.33)$. El programa tuvo una duración de 5 días con 5 horas de clase diarias. El grupo experimental (GE) fue equipado con esquís un $25 \%$ más cortos que el grupo de control (GC). Todos los profesores de esquí siguieron el mismo protocolo de enseñanza. Se grabó a todos los participantes en una bajada en pista verde y un equipo de expertos puntuó la ejecución técnica (VP), el control de la velocidad (CV), control de trayectoria (CT) y el equilibrio (E). El GE obtuvo mejor puntuación en CV , CT y VP respecto al $\mathrm{GC}$, pero sólo la variable VP resultó ser significativa $(\mathrm{M}=1.87$; $\mathrm{DT}=0.76$ vs $\mathrm{M}=1.29$; $\mathrm{DT}=0.58$ ). Se encontraron correlaciones positivas fuertes entre las variables CV y CT $(r=0.80)$, y entre EQ y VP ( $r=0.80)$, y moderadas entre CE y CT $(r=0,66)$, del GE. El empleo de esquís cortos facilita un aprendizaje más eficaz y seguro, pudiendo evolucionar más rápidamente, lo que debe ser tomado en consideración por parte de todos los involucrados en la enseñanza del esquí alpino en edad escolar
\end{abstract}

PALABRAS CLAVE: esquí alpino; deporte escolar; metodología aprendizaje; aprendizaje motor; hábitos saludables. 


\title{
Methodological proposal of initiation to alpine skiing in school children through short skis
}

\begin{abstract}
Alpine skiing is a complex sport but with a low dropout rate, ideal for promoting sports practice at school age. Avoiding failure and frustration at initiation requires a teaching method that facilitates progression. The objective of this study was to determine if a method based on the use of short skis is effective. The sample consisted of 37 subjects, between 8 and 9 years of age ( $M=8.12 ; D T=0.33$ ). The program lasted 5 days with 5 hours of class daily. The experimental group (GE) was equipped with skis $25 \%$ shorter than the control group (GC). All ski teachers followed the same teaching protocol. All participants were recorded on a green slope run and a team of experts scored technical execution (VP), speed control (CV), trajectory control (CT) and balance (E). The GE obtained a better score in CV, CT and VP compared to the GC, but only the variable VP was significant (M = 1.87; DT = 0.76 vs $M=1.29$; $D T=0.58)$. Strong positive correlations were found between the $C V$ and $C T$ variables $(r=0.80)$, and between EQ and VP $(r=0.80)$, and moderate between CE and CT $(r=0.66)$, of the GE. The use of short skis facilitates a more efficient and safe learning process, being able to evolve more quickly, which must be taken into consideration by all professionals in the teaching of alpine skiing at school age.
\end{abstract}

KEYWORDS: alpine skiing; scholar sport; learning methodology; motor skills learning; healthy habits.

\section{Introducción}

El esquí alpino es un deporte de naturaleza que se practica en la montaña, sobre una superficie nevada, y que consiste en deslizarse realizando cambios de dirección sobre dos tablas, los esquís, que otorgan su nombre a esta disciplina deportiva. De procedencia nórdica, es uno de los deportes más antiguos que existen, pues tiene un origen que se remonta a más de 6.000 años atrás. Su función entonces era meramente utilitaria: desplazarse y sobrevivir en terrenos nevados, permitiendo la caza invernal y la defensa (Allen, 2007). Si bien el esquí alpino siempre se ha considerado un deporte minoritario y elitista, su práctica se popularizó a partir de la década de los 80 del siglo pasado, coincidiendo con el desarrollo del turismo, la concienciación sobre la conservación y disfrute de la naturaleza, y los beneficios de la práctica deportiva en la misma, constituyéndose en nuestra sociedad junto con otros deportes practicados en la naturaleza como un modelo alternativo al deporte tradicional (García et al., 2019). Actualmente, y desde el punto de vista recreativo, el esquí alpino es uno de los deportes de invierno más populares, contando con más de 400 millones de practicantes a nivel mundial (Cigrovski, Radman, Konter, Očić, y Ružić, 2018; Vanat, 2018). Huguet i Parellada (1992) definió el esquí como "uno de los deportes más completos que existen, ya que hace trabajar al cuerpo de forma general y 
combina diversos elementos como son la habilidad, la resistencia, la velocidad y la destreza. Además, se practica al aire libre, sin contaminación y en estrecho contacto con la naturaleza, el sol, el frio, la altura..., lo que proporciona salud y vitalidad". Autores como Joksimović, Joksimović, y Acimović (2012) lo definen como "control de la velocidad y de la dirección o trayectorias, completada por la habilidad del equilibrio dinámico en curvas gracias a una buena centralidad de los ejes tobillos, rodillas y cadera”.

Por lo tanto, el esquí alpino es también un deporte complejo. Asociado en su iniciación con la manifestación de ansiedad debido a la incertidumbre del aprendizaje de habilidades y destrezas físicas con riesgo físico (Román, 2008), en el mismo se dan muchos factores, tanto internos como externos, con los que el esquiador debe interactuar; los factores internos son aquellos que resultan de las variables fisiológicas y psicológicas, como la fuerza muscular, o la ansiedad; los externos incluyen el estado de la nieve, las condiciones meteorológicas, la pendiente de la pista, la equipación empleada, la velocidad al esquiar o el radio de viraje empleado. (Dickson, Waddington, y Terwiel, 2018; Seifert, Stöggl, Scheiber, Heizinger, y Müller, 2017). Esta complejidad le confiere a este deporte cierta dificultad para captar nuevos usuarios, pero por otro lado tiene una tasa de abandono muy baja, por lo que puede ser usado como actividad para promover la mejora y prevención de la de salud en cualquier etapa de la vida, cumpliendo además con todas las recomendaciones de práctica deportiva del American College of Sport Medecine y la American Heart Association (Nelson et al., 2007; Supej, Ogrin, y Holmberg, 2018). Para ello, es necesario facilitar y acelerar la progresión por las diferentes fases de la etapa de iniciación al esqui hasta alcanzar un nivel técnico que permita cierta independencia y disfrutar con seguridad en pistas catalogadas como verdes o fáciles, pues esto permite lograr una mayor satisfacción personal que se traducirá en adherencia a la práctica deportiva en el futuro.

La inclusión del esquí alpino en el contexto escolar no sólo ayuda a consolidar la práctica de actividad física y deportiva como la mejor y más saludable opción de ocupar el tiempo de ocio. Desarrolla también un amplio repertorio motriz, posibilitando el incremento de las habilidades básicas y específicas que serán necesarias para el enriquecimiento de la competencia motriz; además, como actividad en la naturaleza que es, permite desenvolverse en la misma con seguridad y con el mayor respeto hacia su conservación (Román-Alconchel, 2008). Los programas escolares de esquí, también llamados "semana blanca", están diseñados para el fomento y popularización de esta actividad deportiva, facilitando a precios muy económicos la práctica y el aprendizaje del esquí alpino en el trascurso de cinco días consecutivos, durante los cuales los alumnos cambian la rutina habitual del centro escolar por clases de esquí en sesiones de cinco horas diarias. En tan concentrado tiempo de práctica, el objetivo principal es conseguir que el alumnado se sienta competente y capaz de bajar por una pista verde encadenando las curvas. Una rápida evolución no sólo va a depender de las habilidades motrices y coordinativas de los alumnos. En el proceso de aprendizaje van a influir otras variables como son el método de enseñanza empleado por el profesorado o la selección correcta del material de 
esquí. Para alcanzar un resultado óptimo, que evite la desmotivación y frustración, el método de enseñanza debe estar correctamente estructurado, debiendo ser éste progresivo y gradual (Lesnik, Žvan, Leskosek, y Supej, 2013).

En nuestro país, al igual que en el resto de países europeos, este método está ya bien consolidado y consensuado, pues para poder ejercer la docencia en el esquí se debe estar en posesión de como mínimo el primer nivel de la titulación de técnico deportivo en esta especialidad, cuyo currículo está especificado en el Real Decreto 319/2000 del Ministerio de Educación y Cultura. Éste incluye el módulo de "metodología de la enseñanza del esquí alpino", cuyos principales contenidos son el aprendizaje de los principios fundamentales de la didáctica y estilos de enseñanza a aplicar en el proceso de enseñanza - aprendizaje, las progresiones metodológicas, y los contenidos técnico-tácticos propios de la etapa de iniciación. Por lo tanto, más allá del estilo propio de enseñanza, no hay grandes variaciones en la metodología empleada por cada profesor de esquí, siendo lo habitual seguir la indicada en la tabla siguiente:

Tabla 1

Progresión metodológica en la iniciación al esqui alpino. Adaptado de Rodríguez y Guerrero (2013)

\begin{tabular}{cl}
\hline Fase & \multicolumn{1}{c}{ Contenido } \\
\hline $1^{\mathrm{a}}$ & $\begin{array}{l}\text { Familiarización entorno, presentación del } \\
\text { material }\end{array}$ \\
\hline $2^{\mathrm{a}}$ & Desplazamiento en llano, descenso directo \\
\hline $3^{\mathrm{a}}$ & $\begin{array}{l}\text { Cuña de frenado, cuña deslizante, virajes en } \\
\text { cuña }\end{array}$ \\
\hline $4^{\mathrm{a}}$ & Stem, viraje fundamental básico y dinámico \\
\hline $5^{\mathrm{a}}$ & Paralelo básico \\
\hline
\end{tabular}

Por otra parte, desde el inicio de la práctica del esquí ha habido una búsqueda del método ideal de aprendizaje basado en modificaciones del material, concretamente de la longitud de los esquís. Es el caso de la propuesta del "esquí evolutivo" de Gruneberg y Blanc (1971), quienes abogaron por el uso de esquís cortos en la iniciación y el incremento progresivo del tamaño de estos en estadios posteriores. De forma análoga, Roschinsky (2004) sugirió el empleo del "método de longitud gradual", creado por Twardokens en Estados Unidos. Estas aportaciones no están infundadas. Buszard, Farrow, Reid, y Masters (2014) afirman que la adaptación del equipamiento en el deporte infantil es beneficiosa para la adquisición de habilidades motoras. Estos beneficios incluyen una mayor participación y disfrute de la tarea, un mejor rendimiento de las habilidades, mejoras rápidas de las habilidades y un desarrollo de patrones de movimiento más deseables y mayor probabilidad de aprender de forma implícita. En general, los defensores del marco basado en restricciones de material argumentan que reducir el tamaño simplifica una habilidad, lo que puede estimular un modo de aprendizaje subconsciente, al permitir que los niños busquen la solución 
más óptima cuando practican (Buszard, Reid, Masters, y Farrow, 2016). Asimismo, la reducción del equipamiento en esquí alpino podría estar en línea con algunos métodos de aprendizaje como es la enseñanza por analogía, dirigida hacia los aspectos globales del movimiento, o la enseñanza con foco externo de atención, focalizada hacia los resultados de la acción en el entorno, terreno y nieve, en lugar de hacia el foco de atención interno, como son los movimientos del cuerpo o en cómo se ejecuta la acción (Van Der Kamp, Duivenvoorden, Kok, y Van Hilvoorde, 2015). Por su parte, Ropret (2014) apostilla que es importante elevar las habilidades a un nivel superior como un primer paso para disminuir el número y la gravedad de las lesiones mientras se practica el esquí. Junto a las aportaciones de este autor sobre la importancia de la educación en competencias técnicas y el conocimiento sobre el uso del equipamiento, destaca también que la reducción del tamaño de los esquís puede facilitar la adquisición de habilidades básicas en esquí al tener una mayor maniobrabilidad en los pies y naturalidad en los gestos por transferencia desde la bipedestación.

Este trabajo tiene como objetivo verificar con datos científicos si una metodología de enseñanza del esquí alpino basada en la reducción del tamaño de los esquís en los primeros estadios puede actuar de mecanismo facilitador de la familiarización al medio nevado.

\section{Método}

\section{Muestra}

La investigación se realizó en el contexto de la semana blanca con alumnado de tercer curso de educación primaria de un centro concertado de la ciudad de Granada. Participaron un total de 37 sujetos, siendo el 54.04\% hombres $(n=20)$ y el $45.94 \%$ mujeres $(n=17)$, de entre 8 y 9 años de edad $(\mathrm{M}=8.12$; $\mathrm{DT}=0.33)$. Todos ellos afirmaron no haber esquiado con anterioridad, siendo por tanto su primer contacto con este deporte. Asistieron de forma voluntaria y con el consentimiento expreso de los padres, quienes fueron informados de la realización del presente estudio, en concordancia con la Declaración de Helsinki de 1975 y las normas éticas del Comité de Investigación, respetándose el resguardo a la confidencialidad de los participantes (Ley 15/1999 del 13 de diciembre).

Tabla 2

Datos muestrales

\begin{tabular}{cccccc}
\hline & Hombres & \multicolumn{2}{l}{ Mujeres } & $\begin{array}{c}\text { Total } \\
\text { muestra }\end{array}$ \\
\hline $\begin{array}{c}\text { Tipo de } \\
\text { esquí }\end{array}$ & $\mathrm{n}$ & $\%$ & $\mathrm{n}$ & $\%$ & $\%$ \\
\hline Esquí corto & 8 & 21.62 & 8 & 21.62 & 43.24 \\
\hline $\begin{array}{c}\text { Esquí } \\
\text { estándar }\end{array}$ & 12 & 32.42 & 9 & 24.32 & 56.76 \\
\hline $\begin{array}{c}\text { Total } \\
\text { muestra }\end{array}$ & 20 & 54.04 & 17 & 45.94 & 100 \\
\hline
\end{tabular}


Diseño del estudio

En este estudió se utilizó un diseño de carácter cuantitativo descriptivo y de tipo transversal. El alumnado se le tomaron las medidas antropométricas (peso y altura) durante las clases de educación física en la semana previa al desarrollo del programa de nieve. El primer dia, antes de comenzar, fueron equipados con material alquilado. Al grupo experimental se le asignaron esquís de tamaño reducido, de longitud entre 80 y $90 \mathrm{~cm}$., lo que representa una disminución del $25 \%$ respecto a la longitud empleada por el grupo de control, el cual utilizó esquís de entre 110 y 120 cm., medida considerada estándar para niños principiantes de las características indicadas en la tabla 4.

El programa de esquí se desarrolló durante cinco días consecutivos, recibiendo todo el alumnado cinco horas diarias de clases prácticas grupales en la estación de Sierra Nevada (Granada). Dado el carácter ecológico del estudio, y por mantener la motivación hacia la práctica, se hicieron 5 grupos mixtos de 6-8 alumnos, donde al menos 2 de los componentes usaron esquís cortos. Debido a las limitaciones de disponibilidad de material, el número de sujetos con esquís más cortos fue algo más reducido que el de los esquís estándar (tabla 3).

Todo el profesorado de esquí seleccionado tenía una experiencia previa de mínimo diez años y la titulación de técnico deportivo de grado medio o superior. Previamente y con la participación de todos, se consensuaron los contenidos técnicos a impartir cada dia, de manera que no hubiera diferencias entre grupos y se siguiera la progresión indicada en la tabla 1.

\section{Variables}

La evaluación de la técnica y destrezas adquiridas se realizó mediante la ejecución de una bajada por pista verde haciendo giros de radio medio alrededor de puntos marcados por balizas, de manera que todo el alumnado ejecutara el mismo número de cambios de dirección y del mismo radio. La prueba fue grabada con dos cámaras de video desde el lateral de la pista y la zona inferior, evitando así puntos ciegos y poder evaluar los movimientos desde el plano frontal y sagital. Posteriormente, un equipo de tres expertos con la titulación de Técnico Deportivo Superior visualizó las grabaciones, valorando y puntuando del 1 al 4 las variables equilibrio, control de la velocidad, control de la trayectoria y la técnica del viraje en paralelo ( $1=$ no lo hace $/$ mal, $2=$ solo en algunos momentos $/$ regular, 3=casi siempre/bien, 4=en todo momento/muy bien), obteniéndose el valor medio de cada una de ellas (tabla 3).

\section{Análisis de los datos}

Para el registro de los datos antropométricos se utilizó una báscula Tanita BC 418 y un tallimetro Seca; para las grabaciones de video dos 
cámaras Sony HDR 405. Todo el análisis estadístico se realizó a través del software R Studio, versión 1.1.463, empleando para los descriptivos básicos medias y frecuencias; para las diferencias de medias entre variables la prueba $\mathrm{T}$ de Student. La correlación de las variables se realizó mediante la prueba de Spearman.

\section{Resultados}

Comenzando con el análisis descriptivo, se constata la homogeneidad de la muestra (tabla 3), obteniéndose similares valores en peso y altura en el grupo de control (GC) y el grupo experimental (GE).

Tabla 3

Caracteristicas antropométricas de la muestra por tipo de esqui

\begin{tabular}{ccccccccc}
\hline $\begin{array}{c}\text { Tipo de } \\
\text { esquí }\end{array}$ & $\begin{array}{c}\text { Hombres } \\
(\mathrm{n})\end{array}$ & $\begin{array}{c}\text { Mujeres } \\
(\mathrm{n})\end{array}$ & $\begin{array}{c}\text { Peso } \\
(\mathrm{M})\end{array}$ & $\begin{array}{c}\text { DT } \\
\text { Esquís }\end{array}$ & $\begin{array}{c}\text { Altura } \\
(\mathrm{M})\end{array}$ & DT & $\begin{array}{c}\text { Tota } \\
1\end{array}$ & $\%$ \\
\hline $\begin{array}{c}\text { cortos } \\
\text { Esquís }\end{array}$ & 8 & 8 & 32.59 & $\begin{array}{c}8.9 \\
3\end{array}$ & 138.53 & $\begin{array}{c}11.0 \\
6\end{array}$ & $\begin{array}{c}\mathrm{n}=1 \\
6\end{array}$ & $\begin{array}{c}43.2 \\
4\end{array}$ \\
\hline $\begin{array}{c}\text { estándar } \\
\mathrm{N}\end{array}$ & 12 & 9 & 35.54 & $\begin{array}{c}9.5 \\
4\end{array}$ & 136.92 & 9.88 & $\begin{array}{c}\mathrm{n}=2 \\
1\end{array}$ & $\begin{array}{c}56.7 \\
6\end{array}$ \\
\hline
\end{tabular}

En la tabla 4 se reflejan los resultados registrados para cada variable. Los valores medios obtenidos por el grupo experimental son más altos para las variables control de velocidad ( $\mathrm{CV} ; \mathrm{M}=2.59$; $\mathrm{DT}=0.73$ ), control de trayectoria (CT; $\mathrm{M}=2.59 ; \mathrm{DT}=0.69$ ) y técnica del viraje paralelo (VP; $\mathrm{M}=1.87 ; \mathrm{DT}=0.76$ ), mientras la variable equilibrio (EQ) es mayor en el grupo de control $(\mathrm{M}=2.40$; $\mathrm{DT}=0.37$. Ninguna de ésta diferencias es estadisticamente significativa, salvo para la variable VP $(\mathrm{p}=0.01)$.

Tabla 4

Resultados de las variables estudiadas por tipo de esqui

\begin{tabular}{ccccccccc}
\hline \multirow{2}{*}{$\begin{array}{c}\text { Tipo } \\
\text { esquís }\end{array}$} & \multicolumn{2}{c}{ Equilibrio } & \multicolumn{2}{c}{$\begin{array}{c}\text { Control } \\
\text { velocidad }\end{array}$} & \multicolumn{2}{c}{$\begin{array}{c}\text { Control } \\
\text { trayectoria }\end{array}$} & \multicolumn{2}{c}{$\begin{array}{c}\text { Técnica viraje } \\
\text { paralelo }\end{array}$} \\
\cline { 2 - 9 } & Media & DT & Media & DT & Media & DT & Media & DT \\
\hline Cortos & 2.37 & 0.46 & 2.59 & 0.73 & 2.59 & 0.69 & 1.87 & 0.76 \\
\hline Largos & 2.40 & 0.37 & 2.43 & 0.43 & 2.36 & 0.48 & 1.29 & 0.58 \\
\hline $\begin{array}{c}\text { Sig. } \\
(95 \%)\end{array}$ & $\mathrm{p}=0,83$ & $\mathrm{p}=0.43$ & $\mathrm{p}=0.25$ & $\mathrm{p}=0.01$ \\
\hline
\end{tabular}


El análisis de las correlaciones entre variables para ambos grupos fue realizado mediante el método Spearman (tablas 5 y 6) y muestra que para el grupo de control no existe correlación entre éstas, pero contrariamente, sí que existe correlación entre CV y CT $(\mathrm{r}=0.80)$, CT y EQ ( $\mathrm{r}=0.66)$ y EQ y VP $(\mathrm{r}=0.80)$ del grupo experimental.

Tabla 5

Correlaciones entre variables para esqui cortos

\begin{tabular}{lcccc}
\hline Variables & $\begin{array}{c}\text { Control } \\
\text { velocida } \\
\mathrm{d}\end{array}$ & $\begin{array}{c}\text { Control } \\
\text { trayectori } \\
\mathrm{a}\end{array}$ & $\begin{array}{c}\text { Equilibr } \\
\text { io }\end{array}$ & $\begin{array}{c}\text { Progresión } \\
\text { viraje } \\
\text { paralelo }\end{array}$ \\
\hline $\begin{array}{l}\text { Control } \\
\text { velocidad }\end{array}$ & & 0.80 & 0.47 & 0.44 \\
\hline $\begin{array}{l}\text { Control } \\
\text { trayectoria }\end{array}$ & & & 0.66 & 0.72 \\
\hline Equilibrio & & & & 0.80 \\
\hline
\end{tabular}

Tabla 6

Correlaciones entre variables para esquís estándar

\begin{tabular}{lcccc}
\hline \multicolumn{1}{c}{ Variables } & $\begin{array}{c}\text { Control } \\
\text { velocida } \\
\text { d }\end{array}$ & $\begin{array}{c}\text { Control } \\
\text { trayectori } \\
\text { a }\end{array}$ & $\begin{array}{c}\text { Equilibr } \\
\text { io }\end{array}$ & $\begin{array}{c}\text { Progresión } \\
\text { viraje } \\
\text { paralelo }\end{array}$ \\
\hline $\begin{array}{l}\text { Control } \\
\text { velocidad }\end{array}$ & & 0.44 & 0.03 & 0.14 \\
\hline $\begin{array}{l}\text { Control } \\
\text { trayectoria }\end{array}$ & & & 0.34 & 0.06 \\
\hline Equilibrio & & & & -0.1 \\
\hline
\end{tabular}

\section{Discusión}

La literatura científica sobre aprendizaje del esquí alpino es muy escasa. El tamaño de la muestra empleada ya refleja las dificultades que representa para la investigación este deporte, especialmente en niños. E1 esquí alpino es estacional, de elevado coste y su práctica organizada dentro del horario escolar habitual impide al alumnado asistir a las clases ordinarias, de ahí que aunque la propuesta de participar en la Semana Blanca se extendiera a las dos líneas de 3er curso de primaria, de los 50 alumnos potenciales finalmente sólo se pudiese contar con la muestra descrita en la tabla 2. Kurpiers y Kerstin (2017) utilizaron una muestra de similar tamaño y edad en su propuesta de aprendizaje del esquí con un solo esquí. Por su parte, Mladenovic et al (2015), en otro estudio con escolares analizaron la mejora de las capacidades motoras mediante el aprendizaje del esquí en una muestra tan sólo algo mayor.

El viraje paralelo básico ya ha sido empleado con éxito en otros 
estudios para evaluar el nivel general de esquí (Cigrovski, Franjko, Ruppic, y Bakovic, 2016; Kurpiers y Kersting, 2017; Mladenović, Cigrovski, Stanković, Prlenda, y Uljević, 2015), y su grado de dominio puede determinar el nivel de destreza en niveles superiores en el futuro, por lo que es importante facilitar su rápido y correcto aprendizaje en las etapas más tempranas (Cigrovski et al., 2016). Según los datos obtenidos en el presente estudio, el empleo de esquis cortos ayuda al alcanzar dicho objetivo, favoreciendo una evolución más rápida a la técnica del viraje en paralelo en comparación con los esquís de longitud estandar, así como un mayor control de la velocidad y trayectoria, variables que están correlacionadas entre sí. Son varias las razones que podrian explicar este hallazgo. Por un lado, el esquí corto facilita la realización de la técnica de la cuña, empleada inicialmente para frenar y girar. Esta técnica aporta estabilidad corporal y facilidad de pivotaje del esquí, debido a su menor peso y menor fricción con la nieve, resultando más sencillo controlar la velocidad (Cigrovski, Prlenda, y Radman, 2014), asi como orientar los esquís hacia la nueva dirección. Por otro lado, ayuda a realizar las acciones corporales necesarias para posicionar de forma graduar los esquí en paralelo (Kurpiers y Kersting, 2017). Otro factor explicativo, en consonancia con los hallazgos de Cigrovski et al. (Cigrovski et al., 2018), se basa en la reducción del miedo a las caidas y lesiones que el mayor control de velocidad puede favorecer, puesto que son superiores en el principiante en el esquí alpino (Ekeland, Rødven, y Heir, 2019).

Los autores Kurpiers y Kersting (2017) investigaron la eficacia de un método de aprendizaje basado tambien en modificaciones del material, empleando sólo un esquí y una pértiga de apoyo, omitiendo la fase de frenado y giro en cuña, con resultados positivos en el aprendizaje final de la tecnica del viraje en paralelo. Sin embargo, los autores Cigrovski, Matković, y Matković (2010), tras comparar el método tradicional de aprendizaje mediante la cuña con otro omitiéndolo, obtuvo puntuaciones más altas en la ejecución del viraje en paralelo en el grupo de control respecto al grupo experimental, concluyendo que no es conveniente eliminar esta fase en el aprendizaje del esquí, conclusión análoga a la obtenida posteriormente por Cigroski et al. (2016) y por Joksimovic et al. (2012).

Según el estudio reciente de Mladenovic et al. (2015), existe correlación entre el aprendizaje de la técnica del esquí alpino en niños de $7 / 8$ años y ciertas cualidades motoras, como la fuerza muscular, estabilidad del tronco, velocidad de movimiento o coordinación. En contraposición, a pesar de la homogeneidad de la muestra empleada en este estudio, los resultados hallados podrian deberse a las diferencias en las cualidades motrices de los sujetos, lo que supone una limitación del mismo, por lo que futuros estudios deberán contemplar la evaluación previa de estos parámetros físicos.

\section{Conclusiones}

Este estudio, según se ha podido comprobar, es el primero hasta la fecha en demostrar científicamente la eficacia del empleo de esquís cortos en 
la iniciación al esquí alpino en población escolar. La importancia de esta investigación radica en su aplicación práctica, pues el empleo de esquís cortos facilita un aprendizaje más eficaz y seguro, pudiendo en un tiempo menor evolucionar a estadios técnicos superiores, evitando el abandono y frustración propios de la iniciación deportiva en los deportes complejos como es el esquí alpino. Esto debe ser tomado en consideración por parte de los responsables de la enseñanza del esquí alpino, clubes deportivos, y por la industria del esquí en general, al poder facilitar la iniciación en este deporte, y con ello, a alcanzar mayores cotas de adherencia a la práctica futura del mismo.

Finalmente, para poder generalizar los hallazgos de esta investigación sería conveniente ampliar el tamaño la muestra, así como realizar un estudio longitudinal para comprobar la eficacia del método propuesto.

\section{Bibliografia}

Allen, E. (2007). The culture and sport of skiing: from antiquity to World War II. University of Massachusetts Press.

Buszard, T., Farrow, D., Reid, M., y Masters, R. (2014). Scaling sporting equipment for children promotes implicit processes during performance. Consciousness and Cognition, 30, 247-255. https://doi.org/10.1016/j.concog.2014.07.004

Buszard, T., Reid, M., Masters, R., y Farrow, D. (2016). Scaling tennis racquets during $\mathrm{PE}$ in primary school to enhance motor skill acquisition. Research quarterly for exercise and sport, 87(4), 414-420. https://doi.org/10.1080/02701367.2016.1216653

Cigrovski, V., Franjko, I., Ruppic, T., y Bakovic, M. (2016). Correlation between balance, specific alpine skiing knowledge and situational efficiency in alpine skiing. Acta Kinesiologica 10 (1), 66-77.

Cigrovski, V., Matković, B., y Matković, B. (2010). Can we make the alpine ski learning more efficient by omitting the snow-plough technique? Sportlogia, 6(2), 51-58. https://doi.org/10.5550/sgia.1002051

Cigrovski, V., Prlenda, N., y Radman, I. (2014). Future of alpine skiing schools-gender related programs. Monten. J. Sports Sci. Med. 3 (1), 5-8.

Cigrovski, V., Radman, I., Konter, E., Očić, M., y Ružić, L. (2018). Sport courage, worry and fear in relation to success of alpine ski learning. Sports, 6(3), 96. https://doi.org/10.3390/sports6030096

Dickson, T. J., Waddington, G., y Terwiel, F. A. (2018). Snowsport experience, expertise, lower limb injury and somatosensory ability. Journal of science and medicine in sport, 22, S17-S21. https://doi.org/10.1016/j.jsams.2018.08.005

Ekeland, A., Rødven, A., y Heir, S. (2019). Injuries among children and adults in alpine skiing and snowboarding. Journal of science and medicine in sport, 22, S3-S6. https://doi.org/10.1016/j.jsams.2018.07.011

López, S., Maneiro, R., Moral, J.E., Amatria, M., Diez, P., Barcala, R. y Abelairas, C. (2019). Los riesgos en la práctica de actividades en la naturaleza . La accidentabilidad en las prácticas deportivas y medidas 
preventivas. Retos (36), 618-624.

Gruneberg, P., y Blanc, R. (1971). Esquiar en tres dias. Barcelona: Editorial Hispano Europea.

Huguet i Parellada, J. (1992). Esqui. Barcelona: Editorial 92 SA.

Joksimović, D., Joksimović, A., y Acimović, D. (2012). Effectivness of two methods in learning basic elements of ski technique. Research in kinesiology, 40(1), 77-81.

Kurpiers, N., y Kersting, U. G. (2017). The one-ski-method-effects of an alternative teaching approach on selected movement patterns in alpine skiing. Cogent Social Sciences, 3(1). https://doi.org/10.1080/23311886.2016.1275958

Lesnik, B., Žvan, M., Leskosek, B., y Supej, M. (2013). Progressivity of basic elements of the slovenian national alpine ski school. Acta Universitatis Carolinae Kinanthropologica, 49(1), 77-93.

Mladenović, D., Cigrovski, V., Stanković, V., Prlenda, N., y Uljević, O. (2015). Success in adopting technique of alpine skiing with respect to motor abilities of the children aged 7-8 Years. Collegium Antropologicum, 39 (1) 77-82.

Nelson, M. E., Rejeski, W. J., Blair, S. N., Duncan, P. W., Judge, J. O., King, A. C., Macera C.A., Castaneda-Sceppa, C. (2007). Physical activity and public health in older adults: recommendation from the American College of Sports Medicine and the American Heart Association. Medicine and Science in Sports and Exercise, 39(8), 1435-1445. https://doi.org/10.1249/mss.0b013e3180616aa2

$\mathrm{R}$ Core Team (2018). R: A language and environment for statisticalcomputing. R Foundation for Statistical Computing, Vienna, Austria. https://www.R-project.org/.

Rodriguez, E., y Guerrero, C. (2013). Aprender a esquiar. Una puerta abierta al mundo de la nieve. Orense: Safe Educación y Formación.

Román Alconchel, B. (2008). La transferencia del patinaje en línea al aprendizaje del esqui alpino en la educación fisica escolar. (Tesis doctoral). Universidad de Granada.

Ropret, R. (2014). Skiing and snowboarding injuries: legal regulations and safety at ski resorts in Serbia. Fizicka kultura 68(2), 178-189.

Roschinsky, J. (2004). Carving. fascination on skis. United Kingdom: Meyer \& Meyer Sport.

Seifert, J., Stöggl, T., Scheiber, P., Heizinger, E., y Müller, E. (2017). Grade and speed have greater influence on HR and RPE than ability, sex, and age in alpine skiing. Journal of Sports Sciences, 35(5), 419-425. https://doi.org/10.1080/02640414.2016.1167935

Supej, M., Ogrin, J., y Holmberg, H. C. (2018). Whole-body vibrations associated with alpine skiing: A risk factor for low back pain? Frontiers in Psychiatry, 9. https://doi.org/10.3389/fphys.2018.00204

Van Der Kamp, J., Duivenvoorden, J., Kok, M., y Van Hilvoorde, I. (2015). Motor skill learning in groups: some proposals for applying implicit learning and self-controlled feedback. RICYDE: Revista Internacional de Ciencias del Deporte, 11(39), 33-47. https://doi.org/ 10.5232/ricyde2015.03903 
Javier Conde-Pipó, Blanca Román, Mario Fernández-Aguilera y Félix Zurita

Vanat, L. (2018). 2018 International Report on Snow and Mountain Tourism. Recuperado de http://vanat.ch/RM-world-report-2018.pdf 